\title{
Downregulated miR-383-5p contributes to the proliferation and migration of gastric cancer cells and is associated with poor prognosis
}

\author{
Chao Wei ${ }^{1}$, Jian-Jun Gao ${ }^{\text {Corresp. } 2}$ \\ ${ }^{1}$ Department of General Surgery, the No.967 Hospital of PLA Joint Logistics Support Force, Postgraduate Culture base of Jinzhou Medical University, \\ Dalian, China \\ 2 Department of General Surgery, the No.967 Hospital of PLA Joint Logistics Support Force, Jinzhou Medical University, Dalian, China \\ Corresponding Author: Jian-Jun Gao \\ Email address: gjj1332006@126.com
}

AIM: The study aims to identify differentially expressed microRNAs (DEMs) in gastric cancer (GC) and explore the expression, prognosis and downstream regulation role of miR-383-5p in GC.

METHODS: The GC miRNA-Seq and clinical information were downloaded from Firebrowse which stores integrated data sourced from The Cancer Genome Atlas (TCGA) database. The DEMs were identified with limma package in R software at the cut-off criteria of $\mathrm{P}<0.05$ and $\mid \log 2$ fold change $\mid>1.0(|\log 2 \mathrm{FC}|>1.0)$. The expression of miR-383-5p in GC cell lines and 54 paired GC tissues was measured by quantitative real-time polymerase chain reaction (qRT-PCR). The overall survival curve of miR-383-5p and the association between its expression and clinicopathological features were explored. Wound healing and cell counting kit-8 (CCK-8) assays were performed to investigate the capacity of miR-383-5p in cell proliferation and migration. The downstream target genes were predicted by bioinformatics tools (miRDB, TargetScan and starBase). The consensus target genes were selected for gene functional enrichment analysis by FunRich v3.0 software. The luciferase reporter assay was performed to verify the potential targeting sites of miR-383-5p on LDHA.

RESULTS: 21 down-regulated miRNAs (including miR-383-5p) and 202 up-regulated miRNAs were identified by analyzing GC miRNA-Seq data. Survival analysis found that patients with low miR-383-5p expression had a shorter survival time (median survival time 21.1 months) than those with high expression (46.9 months). The results of qRT-PCR indicated that miR-383-5p was downregulated in GC cell lines and tissues, which was consistent with miRNA-Seq data. The expression of miR-383-5p was significantly associated with tumour size and differentiation grade. Besides, overexpression of miR-383-5p suppressed GC cells proliferation and migration. A total of 49 common target genes of miR-383-5p were obtained by bioinformatics tools and gene functional enrichment analysis showed that these predicted genes participated in PI3K, mTOR, c-MYC, TGF-beta receptor, VEGF/VEGFR and Ecadherin signaling pathways. The data showed that expression of miR-383-5p was negatively correlated with target LDHA ( $r=-0.203)$. Luciferase reporter assay suggested that LDHA was a target of miR-383-5p.

CONCLUSION: The present study concluded that miR-383-5p was downregulated and may act as a tumor suppressor in GC. Furthermore, its target genes were involved in important signaling pathways. It could be a prognostic biomarker and play a vital role in exploring the molecular mechanism of GC.

KEYWORDS: Gastric cancer; Differentially expressed miRNAs; miR-383-5p; Prognosis; LDHA. 
1 Downregulated miR-383-5p contributes to the proliferation and migration of gastric cancer cells

2 and is associated with poor prognosis.

3

4 Chao Wei ${ }^{1}$, Jian-Jun $\mathrm{Gao}^{2}$

5

$6{ }^{1}$ Department of General Surgery, the No.967 Hospital of PLA Joint Logistics Support Force,

7 Postgraduate Culture base of Jinzhou Medical University, Dalian 116011, China;

$8{ }^{2}$ Department of General Surgery, the No.967 Hospital of PLA Joint Logistics Support Force,

9 Jinzhou Medical University, Dalian 116011, China.

10

11 Corresponding Author:

12 Jian-Jun Gao

13 Department of General Surgery, the No.967 Hospital of PLA Joint Logistics Support Force,

14 Jinzhou Medical University, Dalian 116011, China

15 Email address: gjj1332006@126.com

Abstract

AIM: The study aims to identify differentially expressed microRNAs (DEMs) in gastric cancer

(GC) and explore the expression, prognosis and downstream regulation role of miR-383-5p in $\mathrm{GC}$.

METHODS: The GC miRNA-Seq and clinical information were downloaded from Firebrowse which stores integrated data sourced from The Cancer Genome Atlas (TCGA) database. The DEMs were identified with limma package in $\mathrm{R}$ software at the cut-off criteria of $\mathrm{P}<0.05$ and $\mid \log 2$ fold change $\mid>1.0(|\log 2 \mathrm{FC}|>1.0)$. The expression of miR-383-5p in GC cell lines and 54 paired GC tissues was measured by quantitative real-time polymerase chain reaction (qRT-PCR). The overall survival curve of miR-383-5p and the association between its expression and clinicopathological features were explored. Wound healing and cell counting kit-8 (CCK-8) assays were performed to investigate the capacity of miR-383-5p in cell proliferation and migration. The downstream target genes were predicted by bioinformatics tools (miRDB, TargetScan and starBase). The consensus target genes were selected for gene functional enrichment analysis by FunRich v3.0 software. The luciferase reporter assay was performed to verify the potential targeting sites of miR-383-5p on LDHA.

RESULTS: 21 down-regulated miRNAs (including miR-383-5p) and 202 up-regulated miRNAs were identified by analyzing GC miRNA-Seq data. Survival analysis found that patients with low miR-383-5p expression had a shorter survival time (median survival time 21.1 months) than those with high expression (46.9 months). The results of qRT-PCR indicated that miR-383-5p was downregulated in GC cell lines and tissues, which was consistent with miRNA-Seq data. The expression of miR-383-5p was significantly associated with tumour size and differentiation grade. Besides, overexpression of miR-383-5p suppressed GC cells proliferation and migration. A total of 49 common target genes of miR-383-5p were obtained by bioinformatics tools and 
41 gene functional enrichment analysis showed that these predicted genes participated in PI3K,

42

43

44

45

46

47

48

49

50

51

52

53

54

55

56

57

58

59

60

61

62

63

64

65

66

67

68

69

70

71

72

73

74

75

76

77

78

79

80

mTOR, c-MYC, TGF-beta receptor, VEGF/VEGFR and E-cadherin signaling pathways. The data showed that expression of miR-383-5p was negatively correlated with target LDHA ( $r=-$ 0.203). Luciferase reporter assay suggested that LDHA was a target of miR-383-5p.

CONCLUSION: The present study concluded that miR-383-5p was downregulated and may act as a tumor suppressor in GC. Furthermore, its target genes were involved in important signaling pathways. It could be a prognostic biomarker and play a vital role in exploring the molecular mechanism of GC.

KEYWORDS: Gastric cancer; Differentially expressed miRNAs; miR-383-5p; Prognosis; LDHA.

\section{Introduction}

Gastric cancer (GC) is a common malignancy of human digestive system which has high incidence and mortality worldwide(Bray et al. 2018). In China, both the incidence and mortality of GC rank second among malignant neoplasms(Chen et al. 2016). The number of Chinese patients with GC has increased over the years, which seriously threatens the health of people. The majority of Chinese GC patients are diagnosed at advanced stage and have an unsatisfactory 5-year overall survival rate(Correa 2013). Most studies have found that the development of GC is associated with multiple factors, such as irregular diet, genetic and epigenetic influence(Carcas 2014).

MicroRNAs (miRNAs, 20-24 nucleotides in length) are a series of non-coding RNAs and play important roles in the regulation of gene expression at post-transcriptional level(Zhang et al. 2013). Mechanistically, miRNAs negatively regulate gene expression through binding to sites in the 3'-untranslated regions of messenger RNAs(Zen \& Zhang 2012). Over the past years, mounting studies have confirmed that miRNAs can act as oncogenes or anti-oncogenes in the initiation and development of GC by regulating the downstream target genes(Chen et al. 2018; Hui et al. 2018; Wang et al. 2019b). Thus, exploring the expression and regulation role of miRNAs may be in favor of uncovering the tumorigenesis mechanism of GC.

In present study, we first identified that miR-383-5p was down-regulated in GC tissues by analyzing GC miRNA-Seq data. In order to confirm this finding, qRT-PCR experiment was performed to measure the expression of miR-383-5p in GC cell lines and tissues. Kaplan-Meier survival analysis also found that patients in high miR-383-5p expression group have longer overall survival time than those in low miR-383-5p expression group. Overexpression of miR$383-5 p$ suppressed GC cells proliferation and migration. All the results showed that miR-383-5p was downregulated and it may play an anti-oncogene role in GC. The potential target genes of miR-383-5p were predicted through online bioinformatics tools. The functional enrichment analysis of target genes indicated that miR-383-5p may take part in PI3K, mTOR, c-MYC, TGFbeta receptor, VEGF/VEGFR and E-cadherin signaling pathways. LDHA, one of the 49 common target genes of miR-383-5p, was selected for target validation. The data showed that the expression of miR-383-5p was negatively correlated with LDHA ( $r=-0.203)$. Furthermore, luciferase reporter assay suggested that the luciferase activity in wild-type LDHA-3'UTR group

Peer) reviewing PDF | (2019:06:38233:1:2:NEW 14 Aug 2019) 
81 was significantly decreased by the miR-383-5p mimics, and there were no differences in the

82

83

84

85

86

87

88

89

90

91

92

93

94

95

96

97

98

99

100

101

102

103

104

105

106

107

108

109

110

111

112

113

114

115

116

117

118

119

120

mutant LDHA-3'UTR group. Above all, miR-383-5p can be a meaningful target in understanding the potential molecular mechanism of GC tumorigenesis and progression.

\section{Materials \& Methods}

\section{MiRNA-Seq data and clinical information}

The GC clinical information and miRNA-Seq data, which contain 389 cancer tissue samples and 41 gastric normal tissue samples, were downloaded from Firebrowse website (TCGA data version 2016_01_28). At the cut-off criterion of $\mid \log 2$ fold change $\mid>1.0(|\log 2 \mathrm{FC}|>1.0)$ and $\mathrm{P}<$ 0.05 , the DEMs were identified using limma package in $\mathrm{R}$ software. The follow-up days and vital status of patients were extracted from clinical information data. Patients meeting the following criteria were included for overall survival: (1) patients have integrated follow-up days and vital status; (2) patients have both follow-up days and expression value of miR-383-5p. Totally, 382 patients were respectively divided into low and high expression group according to the median value of miR-383-5p expression. The overall survival curve of low and high miR$383-5 p$ expression groups were analyzed with the method of Kaplan-Meier and log-rank test. GC cell lines culture

Human GC cell lines (SGC-7901, BGC-823, MGC-803, and MKN-45) and a normal gastric mucous membrane cell line (GES-1) were purchased from the Institute of Biochemistry and Cell Biology of the Chinese Academy of Sciences (Shanghai, China), and all cell lines were cultivated in RPMI 1640 medium (GIBCO-BRL) supplemented with $10 \%$ fetal bovine serum (FBS; Gibco, Grand Island, NY, USA), $100 \mathrm{U} / \mathrm{mL}$ penicillin and $100 \mathrm{mg} / \mathrm{mL}$ streptomycin. All cells were cultured in humidified air at $37{ }^{\circ} \mathrm{C}$ and $5 \% \mathrm{CO}$.

\section{Patient tissues collection}

All the GC tissues and the corresponding adjacent normal tissues were collected from 54 patients who received surgical resection at the No.967 Hospital of PLA Joint Logistics Support Force and the Northern Theater Command General Hospital. Tissues were histologically confirmed and immediately stored at $-80^{\circ} \mathrm{C}$ after resection. The clinicopathological features of 54 GC patients were recorded and preserved. All patients signed the informed consent (see detail human participant consent form in supplementary file) and this study was approved by the Research Ethics Committee of Jinzhou Medical University (IRB No: EC-2018-JZ-016).

$R N A$ extraction and $q R T-P C R$ assays

The TRIzol reagent (Invitrogen, CA, USA) was utilized for extracting total RNA of tissues and cells. Reverse Transcription Kit (GenePharma, China) was used for obtaining cDNA reverse transcribed from RNA. qRT-PCR assay was performed with SYBR-Green Hairpin-itTM MicroRNAs Kit (GenePharma, China), which was conducted on ABI 7500 FAST Real-Time PCR System. The expression level was determined using 2- $\Delta \Delta \mathrm{Ct}$ method and normalized to U6. All the sequences of primers used in present study were summarized in Table $\mathbf{1 .}$

RNA oligonucleotide and cell transfection

The miR-383-5p mimics and mimics NC were designed and synthesized by GenePharma Co., Ltd (Shanghai, China). According to the manufacturer's protocols, the GC cells were 
121 transfected using Lipofectamine ${ }^{\text {TM }} 3000$ reagent (Invitrogen; Thermo Fisher Scientific.). All the

122 cells were cultivated for $48 \mathrm{~h}$ after transfection.

123 Cell proliferation assay

124 After transfection, the MGC-803 or MKN-45 cells were seeded into a 96-well plate with 4

$125 \times 10^{3}$ cells per well in triplicate. At $0,24,48$, and $72 \mathrm{~h}$, each well was added with $10 \mu \mathrm{L}$ of CCK-

1268 reagent (Dojindo, Japan) and then incubated at $37^{\circ} \mathrm{C}$ for $3 \mathrm{~h}$. The absorbance at $450 \mathrm{~nm}$ was

127 measured using a spectrophotometer.

128 Wound healing assay

129 The MGC-803 or MKN-45 cells were seeded into 6-well plate. When the cells were

130 cultured to a density of $90 \%$, a $100 \mu \mathrm{L}$ pipette tip was used to draw a straight wound. Then, the

131 cells were cultured with serum-free medium in the humidified incubator. At 0 and $48 \mathrm{~h}$, an

132 inverted microscope was utilized to visualize the wound healing and photograph. Before

133 observing the healing status, mitomycin $\mathrm{C}(10 \mathrm{ug} / \mathrm{ml})$ was added into each well for $2 \mathrm{~h}$ to exclude

134 the influence of proliferation on cell migration.

135 Target genes prediction of miR-383-5p and functional enrichment analysis

136 The bioinformatics websites of TargetScan(Agarwal et al. 2015)

137 (http://www.targetscan.org/vert_71/), miRDB (Wang 2016) (http://www.mirdb.org/) and

138 starBase(Li et al. 2014) (http://starbase.sysu.edu.cn/) were applied for predicting potential target

139 genes of miR-383-5p. The consensus results of the three tools were selected for further analysis.

140 Genes functional enrichment analysis was performed by FunRich v3.0 software which was a

141 widely used tool for the gene functional enrichment and interaction network analysis(Pathan et

142 al. 2015). All the procedures were conducted according to official protocols and default

143 parameters.

144 Luciferase reporter assay

145 The luciferase reporter vectors containing wild-type (WT) or mutant (MUT) 3' UTR of

146 LDHA and miR-383-5p mimics or negative control were transfected into MKN-45 cells.

147 Luciferase activity was tested using the Dual-Luciferase Reporter Assay System (Promega,

148 Madison, WI, USA). Activities were normalized to Renilla luciferase. All experiments were

149 performed three times.

150 Statistical analysis

151 MiRNA-Seq data was processed by the limma(Ritchie et al. 2015) package in R software.

152 The survival curve was described by Kaplan-Meier survival plot and analyzed with log-rank test.

153 The differences between the two groups were analyzed by paired or unpaired Student's t-test.

154 The chi-square test was used for exploring the association between miR-383-5p expression and

155 clinical features (such as: age, gender, tumor size, lymph node metastasis, TNM stage, and

156 differentiation grade). $\mathrm{P}<0.05$ was recognized as statistically significant and all statistical

157 analysis were conducted by IBM SPSS software 19.0.

158 Results

159 Identification of DEMs in GC 
160

161

162

163

164

165

166

167

168

169

170

171

172

173

174

175

176

177

178

179

180

181

182

183

184

185

186

187

188

189

190

191

192

193

194

195

196

197

198

199

At the cut-off criterion of $|\log 2 \mathrm{FC}|>1.0$ and $\mathrm{P}<0.05,223$ DEMs were identified by screening GC miRNA-Seq data. A volcano plot was drawn to visualize the 21 down-regulated and 202 up-regulated miRNAs (Figure 1). The top 20 of down- and up-regulated miRNAs ranked by FC was listed in Table 2 . We found that miR-383-5p was significantly downregulated with a $\log 2 \mathrm{FC}$ of -1.12 , which indicated that it may act as a tumor-suppressor in GC. MiR-383-5p was confirmed to be downregulated in $G C$

The expression value of miR-383-5p was extracted from miRNA-Seq data. Totally, there were 387 GC samples and 41 normal samples. The expression of miR-383-5p in GC was significantly lower than normal tissues (Figure 2A). The results of qRT-PCR showed that miR383-5p was significantly down-regulated in GC at the level of cell and tissue (Figure 2 B and 2C). Combining the miRNA-Seq data and qRT-PCR assay, we confirmed that miR-383-5p was down-regulated and might be a novel tumor suppressor gene in GC.

Association between miR-383-5p and prognosis, clinicopathological features

A total of 382 GC TCGA samples with necessary data were selected to investigate the prognostic role of miR-383-5p. According to the median expression value of miR-383-5p, patients were equally divided to the low and high expression groups. The Kaplan-Meier survival analysis indicated that patients with low miR-383-5p expression had a shorter survival time (median survival time 21.1 months) than those with high expression (46.9 months) (Figure 3). Furthermore, we explored the association between miR-383-5p expression and clinicopathological features. The results manifested that low miR-383-5p expression was significantly associated with large tumor size and poor differentiation grade (Table 3 ).

Nevertheless, the features of age, gender, lymph node metastasis and TNM stage were found to be of no significant difference.

Overexpression of miR-383-5p inhibits GC cell proliferation and migration

The CCK-8 and wound healing assays were performed to assess the effect of miR-383-5p on the proliferation and migration of GC cells. Compared with NC group, transfection with miR383-5p mimics weakened the migration capacity of MGC-803 and MKN-45 cells (Figure 4AH). Besides, the CCK-8 assay showed that miR-383-5p mimics inhibited GC cells proliferation (Figure $4 \mathrm{I}$ and $4 \mathbf{J}$ ).

\section{Target prediction and genes functional enrichment analyses}

Three bioinformatics websites (TargetScan, miRDB, and starBase) were selected for predicting target genes of miR-383-5p. In view of that each website had diverse bioinformatics algorithm, we took the consensus results of different predictions. As described in the venn plot, 49 consensus target genes were obtained (Figure 5A). To comprehend the function of miR-383$5 p$ target genes, the 49 genes were used for functional enrichment analysis by FunRich. At the aspect of biological pathway analysis, we found that these genes participated in PI3K, mTOR, cMYC, TGF-beat receptor, VEGF/VEGFR and E-cadherin signaling pathways (Figure 5B). MiR-383-5p targets LDHA

LDHA is one of the 49 common target genes and it has a quite higher target prediction score. The miRNA-target prediction from starBase demonstrated that miR-383-5p had potential 
200 binding site on the 3'UTR of LDHA (Figure 6A). The association regression analysis based on 201 starBase project indicated that miR-383-5p expression was negatively correlated with LDHA 202 (Figure 6B). Furthermore, luciferase reporter assay suggested that the luciferase activity in wild203 type LDHA-3'UTR group was significantly decreased by the miR-383-5p mimics, and there 204 were no differences in the mutant LDHA-3'UTR group (Figure 6C). All the results indicated 205 that LDHA was a target of miR-383-5p.

206 Discussion

207 GC has the malignant features of terrible proliferation, invasion, metastasis and multiple 208 drug resistance, which lead to high mortality and poor prognosis. Increasing studies have proved 209 that miRNAs are aberrantly expressed and involved in the initiation and development of 210 GC(Chen et al. 2019; Kang et al. 2018; Maruyama et al. 2018; Wang et al. 2019a). Thus, 211 identifying DEMs and exploring the biological function of miRNAs can be useful for finding 212 novel biomarkers and understanding the mechanism of GC progression.

213 In this study, we first downloaded and analyzed the GC miRNA-Seq and clinical data from 214 Firebrowse website(Deng et al. 2017), which conserved integrated gene expression profiles and 215 clinical information data from TCGA. Through screening for DEMs, we found that miR-383-5p 216 was down-regulated in GC tissues. Besides, we also investigated the prognostic role of miR-383217 5p, and Kaplan-Meier survival analysis indicated that patients with low miR-383-5p expression 218 had a shorter survival time than those with high expression. All these results inspired us that 219 miR-383-5p may play an important role in GC.

220

221

222

223

224

225

226

227

228

229

230

231

232

233

234

235

236

237

238

239

Furthermore, we retrieved literatures published worldwide to comprehend the studies about miR-383-5p. Zhao et al. found that miR-383-5p was significantly decreased in lung adenocarcinoma and overexpression of miR-383-5p inhibited cell proliferation by G1 cell cycle phase arrest and induced apoptosis in vitro(Zhao et al. 2017). In hepatocellular carcinoma, miR$383-5 \mathrm{p}$ was proved to be a tumor suppressor and to modulate hepatocellular carcinoma tumorigenesis and progress by targeting AKR1B10 (Wang et al. 2018) and LDHA(Fang et al. 2017). Besides, Jiang's study reported that overexpression of miR-383-5p could inhibit ovarian cancer cell proliferation and enhance chemosensitivity of cells by regulating TRIM27 (Jiang et al. 2019). MiR-383-5p could also suppress ovarian cancer cell proliferation, invasion and aerobic glycolysis through regulating LDHA(Han et al. 2017). Azarbarzin et. al found that miR-383-5p was downregulated in intestinal-type GC and could be used a diagnostic biomarker(Azarbarzin et al. 2017). However, the molecular function and clinical significance of miR-383-5p in GC have not been studied. The present study investigated that miR-383-5p was decreased in GC and its expression was associated with tumor size and differentiation grade. Furthermore, the CCK-8 and wound healing assays demonstrated that overexpression of miR-383-5p could inhibit GC cells proliferation and migration. All the results indicated that miR-383-5p could act as a tumor suppressor in GC and it also had vital clinical value.

We further explored the downstream regulation role by predicting the potential target genes of miR-383-5p. The consensus target genes were obtained by integrating the results from three bioinformatics tools, which improved the accuracy of prediction. The functional enrichment 
240 analysis demonstrated that miR-383-5p may be involved in PI3K, mTOR, c-MYC, TGF-beta

241 receptor, VEGF/VEGFR and E-cadherin signaling pathways through regulating the target genes.

242 It is well known that mTOR pathway regulates tumor growth and metastasis by mediating tumor

243 metabolic homeostasis(Xia \& Xu 2015). Multiple miRNAs were reported to participate in the

244 regulation of PI3K/AKT/mTOR signaling pathway(Riquelme et al. 2016). Yu's study showed

245 that miR-106b was overexpressed in CD44(+) GC stem-like cells and could retain cancer stem

246 cell characteristics through modulating TGF- $\beta$ /Smad signaling pathway(Yu et al. 2014). MiR-

247372 negatively targets KIF26B to suppresses GC cells proliferation and metastasis by regulating

248 VEGF pathway(Zhang et al. 2017). Above all, miR-383-5p may act as a novel tumor suppressor

249 in taking part in the biological function of GC.

250 LDHA acts as a glycolytic enzyme in the process of catalyzing the formation of lactate from

251 pyruvate. LDHA is not only involved in normal cells metabolism, but closely related to tumor

252 malignancy. Studies have found that LDHA was upregulated in kinds of malignant tumors and

253 can play an oncogene role in GC (Wang et al. 2017; Zhu et al. 2018). The association regression

254 analysis based on starBase project indicated that miR-383-5p expression was negatively

255 correlated with LDHA. Besides, luciferase reporter assay showed that the luciferase activity in

256 wild-type LDHA-3'UTR group was significantly decreased by the miR-383-5p mimics, and

257 there were no differences in the mutant LDHA-3'UTR group. All the results suggested that

258 LDHA was a downstream target of miR-383-5p.

259 Conclusions

260 In summary, we found that miR-383-5p may act as a tumor suppressor in GC. It is of

261 important clinical significance and prognostic value, which could contribute to revealing the

262 molecular mechanism of GC tumorigenesis and progress.

263 Acknowledgements

264

265

266

267

268

269

270

271

272

273

274

275

276

277

278

279

280

281

\section{References}

Agarwal V, Bell GW, Nam JW, and Bartel DP. 2015. Predicting effective microRNA target sites in mammalian mRNAs. Elife 4. 10.7554/eLife.05005

Azarbarzin S, Feizi MAH, Safaralizadeh R, Kazemzadeh M, and Fateh A. 2017. The Value of MiR-383, an Intronic MiRNA, as a Diagnostic and Prognostic Biomarker in Intestinal-Type Gastric Cancer. Biochem Genet 55:244-252. 10.1007/s10528-017-9793-x

Bray F, Ferlay J, Soerjomataram I, Siegel RL, Torre LA, and Jemal A. 2018. Global cancer statistics 2018: GLOBOCAN estimates of incidence and mortality worldwide for 36 cancers in 185 countries. $C A$ Cancer J Clin 68:394-424. 10.3322/caac.21492 \%/ (c) 2018 American Cancer Society.

Carcas LP. 2014. Gastric cancer review. J Carcinog 13:14. 10.4103/1477-3163.146506

Chen D, Wang H, Chen J, Li Z, Li S, Hu Z, Huang S, Zhao Y, and He X. 2018. MicroRNA-129-5p Regulates Glycolysis and Cell Proliferation by Targeting the Glucose Transporter SLC2A3 in Gastric Cancer Cells. Front Pharmacol 9:502. 10.3389/fphar.2018.00502

Chen W, Zheng R, Baade PD, Zhang S, Zeng H, Bray F, Jemal A, Yu XQ, and He J. 2016. Cancer statistics in China, 2015. CA Cancer J Clin 66:115-132. 10.3322/caac.21338

Chen Z, Li Z, Soutto M, Wang W, Piazuelo MB, Zhu S, Guo Y, Maturana MJ, Corvalan AH, Chen X, Xu Z, and El-Rifai WM. 2019. Integrated Analysis of Mouse and Human Gastric Neoplasms Identifies 
282

283

284

285

286

287

288

289

290

291

292

293

294

295

296

297

298

299

300

301

302

303

304

305

306

307

308

309

310

311

312

313

314

315

316

317

318

319

320

321

322

323

324

325

326

327

328

329

Conserved microRNA Networks in Gastric Carcinogenesis. Gastroenterology 156:1127-1139 e1128. 10.1053/j.gastro.2018.11.052

Correa P. 2013. Gastric cancer: overview. Gastroenterol Clin North Am 42:211-217. 10.1016/j.gtc.2013.01.002 \%/ Copyright (c) 2013 Elsevier Inc. All rights reserved.

Deng M, Bragelmann J, Kryukov I, Saraiva-Agostinho N, and Perner S. 2017. FirebrowseR: an R client to the Broad Institute's Firehose Pipeline. Database (Oxford) 2017. 10.1093/database/baw160

Fang Z, He L, Jia H, Huang Q, Chen D, and Zhang Z. 2017. The miR-383-LDHA axis regulates cell proliferation, invasion and glycolysis in hepatocellular cancer. Iran J Basic Med Sci 20:187-192. 10.22038/ijbms.2017.8246

Han RL, Wang FP, Zhang PA, Zhou XY, and Li Y. 2017. miR-383 inhibits ovarian cancer cell proliferation, invasion and aerobic glycolysis by targeting LDHA. Neoplasma 64:244-252. 10.4149/neo_2017_211

Hui W, Ma X, Zan Y, Song L, Zhang S, and Dong L. 2018. MicroRNA-1292-5p inhibits cell growth, migration and invasion of gastric carcinoma by targeting DEK. Am J Cancer Res 8:1228-1238.

Jiang J, Xie C, Liu Y, Shi Q, and Chen Y. 2019. Up-regulation of miR-383-5p suppresses proliferation and enhances chemosensitivity in ovarian cancer cells by targeting TRIM27. Biomed Pharmacother 109:595-601. 10.1016/j.biopha.2018.10.148

Kang W, Huang T, Zhou Y, Zhang J, Lung RWM, Tong JHM, Chan AWH, Zhang B, Wong CC, Wu F, Dong Y, Wang S, Yang W, Pan Y, Chak WP, Cheung AHK, Pang JCS, Yu J, Cheng ASL, and To KF. 2018. miR-375 is involved in Hippo pathway by targeting YAP1/TEAD4-CTGF axis in gastric carcinogenesis. Cell Death Dis 9:92. 10.1038/s41419-017-0134-0

Li JH, Liu S, Zhou H, Qu LH, and Yang JH. 2014. starBase v2.0: decoding miRNA-ceRNA, miRNAncRNA and protein-RNA interaction networks from large-scale CLIP-Seq data. Nucleic Acids Res 42:D92-97. 10.1093/nar/gkt1248

Maruyama S, Furuya S, Shiraishi K, Shimizu H, Akaike H, Hosomura N, Kawaguchi Y, Amemiya H, Kawaida H, Sudo M, Inoue S, Kono H, and Ichikawa D. 2018. miR-122-5p as a novel biomarker for alpha-fetoprotein-producing gastric cancer. World J Gastrointest Oncol 10:344-350. 10.4251/wjgo.v10.i10.344

Pathan M, Keerthikumar S, Ang CS, Gangoda L, Quek CY, Williamson NA, Mouradov D, Sieber OM, Simpson RJ, Salim A, Bacic A, Hill AF, Stroud DA, Ryan MT, Agbinya JI, Mariadason JM, Burgess AW, and Mathivanan S. 2015. FunRich: An open access standalone functional enrichment and interaction network analysis tool. Proteomics 15:2597-2601. 10.1002/pmic.201400515

Riquelme I, Tapia O, Leal P, Sandoval A, Varga MG, Letelier P, Buchegger K, Bizama C, Espinoza JA, Peek RM, Araya JC, and Roa JC. 2016. miR-101-2, miR-125b-2 and miR-451a act as potential tumor suppressors in gastric cancer through regulation of the PI3K/AKT/mTOR pathway. Cell Oncol (Dordr) 39:23-33. 10.1007/s13402-015-0247-3

Ritchie ME, Phipson B, Wu D, Hu Y, Law CW, Shi W, and Smyth GK. 2015. limma powers differential expression analyses for RNA-sequencing and microarray studies. Nucleic Acids Res 43:e47. 10.1093/nar/gkv007

Wang H, Zhou R, Sun L, Xia J, Yang X, Pan C, Huang N, Shi M, Bin J, Liao Y, and Liao W. 2017. TOP1MT deficiency promotes GC invasion and migration via the enhancements of LDHA expression and aerobic glycolysis. Endocr Relat Cancer 24:565-578. 10.1530/ERC-17-0058

Wang J, Zhou Y, Fei X, Chen X, and Chen Y. 2018. Biostatistics mining associated method identifies AKR1B10 enhancing hepatocellular carcinoma cell growth and degenerated by miR-383-5p. Sci Rep 8:11094. 10.1038/s41598-018-29271-3

Wang R, Sun Y, Yu W, Yan Y, Qiao M, Jiang R, Guan W, and Wang L. 2019a. Downregulation of miRNA214 in cancer-associated fibroblasts contributes to migration and invasion of gastric cancer cells through targeting FGF9 and inducing EMT. J Exp Clin Cancer Res 38:20. 10.1186/s13046-018-0995-9

Peer] reviewing PDF | (2019:06:38233:1:2:NEW 14 Aug 2019) 
330

331

332

333

334

335

336

337

338

339

340

341

342

343

344

345

346

347

348

349

350

351

352

353

354

355

Wang X. 2016. Improving microRNA target prediction by modeling with unambiguously identified microRNA-target pairs from CLIP-ligation studies. Bioinformatics 32:1316-1322. 10.1093/bioinformatics/btw002

Wang YN, Xu F, Zhang P, Wang P, Wei YN, Wu C, and Cheng SJ. 2019b. MicroRNA-575 regulates development of gastric cancer by targeting PTEN. Biomed Pharmacother 113:108716. 10.1016/j.biopha.2019.108716

Xia P, and Xu XY. 2015. PI3K/Akt/mTOR signaling pathway in cancer stem cells: from basic research to clinical application. Am J Cancer Res 5:1602-1609.

Yu D, Shin HS, Lee YS, and Lee YC. 2014. miR-106b modulates cancer stem cell characteristics through TGF-beta/Smad signaling in CD44-positive gastric cancer cells. Lab Invest 94:1370-1381. 10.1038/labinvest.2014.125

Zen K, and Zhang CY. 2012. Circulating microRNAs: a novel class of biomarkers to diagnose and monitor human cancers. Med Res Rev 32:326-348. 10.1002/med.20215

Zhang H, Ma RR, Wang XJ, Su ZX, Chen X, Shi DB, Guo XY, Liu HT, and Gao P. 2017. KIF26B, a novel oncogene, promotes proliferation and metastasis by activating the VEGF pathway in gastric cancer. Oncogene 36:5609-5619. 10.1038/onc.2017.163

Zhang Y, Wang Z, and Gemeinhart RA. 2013. Progress in microRNA delivery. J Control Release 172:962974. 10.1016/j.jconrel.2013.09.015

Zhao S, Gao X, Zang S, Li Y, Feng X, and Yuan X. 2017. MicroRNA-383-5p acts as a prognostic marker and inhibitor of cell proliferation in lung adenocarcinoma by cancerous inhibitor of protein phosphatase 2A. Oncol Lett 14:3573-3579. 10.3892/ol.2017.6603

Zhu W, Ma L, Qian J, Xu J, Xu T, Pang L, Zhou H, Shu Y, and Zhou J. 2018. The molecular mechanism and clinical significance of LDHA in HER2-mediated progression of gastric cancer. Am J Transl Res 10:2055-2067. 
356 Table 1. Sequence of primers used for PCR.

\begin{tabular}{ll}
\hline Name & Sequence $\left(\mathbf{5}^{\prime} \mathbf{-} \mathbf{3}^{\prime} \mathbf{)}\right.$ \\
\hline mR-383-5p (RT) & GTCGTATCCAGTGCGTGTCGTGGAGTCGGCAATTGCACTGG \\
& ATACGACAGCCAC \\
mR-383-5p (forward) & GGGAGATCAGAAGGTGATTGTGGCT \\
mR-383-5p (reverse) & CAGTGCGTGTCGTGGAGT \\
U6 (forward) & CTCGCTTCGGCAGCACA \\
U6 (reverse) & AACGCTTCACGAATTTGCGT \\
\hline
\end{tabular}

Table 242 DEMs identified between GC and adjacent normal tissues

\begin{tabular}{lll|lll}
\hline Down-regulated & logFC & P value & Up-regulated & logFC & P value \\
\hline miR-1-3p & -3.13 & $1.56 \mathrm{E}-19$ & miR-196a-5p & 5.55 & $3.01 \mathrm{E}-39$ \\
miR-133a-3p & -3.13 & $9.66 \mathrm{E}-20$ & miR-196b-5p & 4.94 & $3.33 \mathrm{E}-28$ \\
miR-133b & -3.03 & $9.52 \mathrm{E}-20$ & miR-767-5p & 4.05 & $3.28 \mathrm{E}-06$ \\
miR-802 & -2.46 & 0.000768 & miR-552-3p & 3.55 & 0.000579 \\
miR-490-3p & -2.46 & $2.63 \mathrm{E}-08$ & miR-105-5p & 3.49 & $7.65 \mathrm{E}-06$ \\
miR-1265 & -2.43 & 0.014022 & miR-135b-5p & 3.46 & $2.25 \mathrm{E}-21$ \\
miR-204-5p & -2.10 & $3.67 \mathrm{E}-11$ & miR-767-3p & 3.44 & 0.003757 \\
miR-145-5p & -1.87 & $3.04 \mathrm{E}-17$ & miR-194-5p & 2.97 & $2.20 \mathrm{E}-22$ \\
miR-139-3p & -1.76 & $2.84 \mathrm{E}-21$ & miR-200a-5p & 2.94 & $2.40 \mathrm{E}-23$ \\
miR-145-3p & -1.75 & $2.37 \mathrm{E}-19$ & miR-192-5p & 2.87 & $1.71 \mathrm{E}-18$ \\
miR-129-5p & -1.70 & $4.41 \mathrm{E}-08$ & miR-200a-3p & 2.81 & $2.26 \mathrm{E}-19$ \\
miR-139-5p & -1.59 & $2.49 \mathrm{E}-21$ & miR-200b-3p & 2.80 & $1.08 \mathrm{E}-22$ \\
miR-30a-3p & -1.41 & $4.25 \mathrm{E}-13$ & miR-141-5p & 2.79 & $5.05 \mathrm{E}-23$ \\
miR-490-5p & -1.40 & $6.09 \mathrm{E}-07$ & miR-1269a & 2.78 & 0.003274 \\
miR-551b-3p & -1.32 & $1.73 \mathrm{E}-06$ & miR-183-5p & 2.71 & $4.27 \mathrm{E}-21$ \\
miR-143-3p & -1.27 & $1.05 \mathrm{E}-11$ & miR-194-3p & 2.64 & $1.03 \mathrm{E}-15$ \\
miR-486-5p & -1.22 & $2.70 \mathrm{E}-07$ & miR-429 & 2.58 & $2.15 \mathrm{E}-14$ \\
miR-29c-3p & -1.14 & $7.86 \mathrm{E}-12$ & miR-141-3p & 2.57 & $5.25 \mathrm{E}-17$ \\
miR-383-5p & -1.12 & $1.13 \mathrm{E}-05$ & miR-675-5p & 2.55 & 0.011142 \\
miR-195-3p & -1.06 & $1.83 \mathrm{E}-09$ & miR-146b-5p & 2.50 & $4.41 \mathrm{E}-38$ \\
\hline
\end{tabular}


360 Table 3. Association between miR-383-5p expression and clinical features.

\begin{tabular}{|c|c|c|c|c|c|}
\hline \multirow[b]{2}{*}{ Variables } & \multicolumn{2}{|c|}{ MiR-383-5p expression } & \multirow{2}{*}{$\begin{array}{c}\text { Total } \\
\text { samples }\end{array}$} & \multirow{2}{*}{$\begin{array}{c}\text { Pearson } \\
r\end{array}$} & \multirow[b]{2}{*}{ P value } \\
\hline & Low $(n, \%)$ & $\begin{array}{l}\text { High } \\
(\mathrm{n}, \%)\end{array}$ & & & \\
\hline \multicolumn{6}{|l|}{ Age } \\
\hline$<60$ & $11(17.1)$ & $12(13.4)$ & 23 & \multirow{2}{*}{-0.037} & \multirow{2}{*}{0.783} \\
\hline$\geq 60$ & $16(28.0)$ & $15(41.5)$ & 31 & & \\
\hline \multicolumn{6}{|l|}{ Gender } \\
\hline Male & $17(32.9)$ & $13(28.1)$ & 30 & \multirow{2}{*}{0.149} & \multirow{2}{*}{0.273} \\
\hline Female & $10(25.6)$ & $14(13.4)$ & 24 & & \\
\hline \multicolumn{6}{|l|}{ Tumour size } \\
\hline$\leq 5 \mathrm{~cm}$ & $5(9.8)$ & $14(15.9)$ & 19 & \multirow{2}{*}{-0.349} & \multirow{2}{*}{$0.010^{*}$} \\
\hline$>5 \mathrm{~cm}$ & $22(48.8)$ & $13(25.6)$ & 35 & & \\
\hline \multicolumn{6}{|c|}{ Lymph node metastasis } \\
\hline Negative & $12(13.4)$ & $18(19.5)$ & 30 & \multirow{2}{*}{-0.224} & \multirow{2}{*}{0.100} \\
\hline Positive & $15(45.1)$ & $9(22.0)$ & 24 & & \\
\hline \multicolumn{6}{|l|}{ TNM stage } \\
\hline $\mathrm{I}+\mathrm{II}$ & $13(28.0)$ & $20(15.9)$ & 33 & \multirow{2}{*}{-0.266} & \multirow{2}{*}{0.051} \\
\hline $\mathrm{III}+\mathrm{IV}$ & $14(30.5)$ & $7(25.6)$ & 21 & & \\
\hline \multicolumn{6}{|c|}{ Differentiation grade } \\
\hline Well and moderate & $6(17.1)$ & $16(18.3)$ & 22 & \multirow{2}{*}{-0.377} & \multirow{2}{*}{$0.006^{*}$} \\
\hline Poor & $21(41.5)$ & $11(23.2)$ & 32 & & \\
\hline
\end{tabular}

$* \mathrm{P}<0.05$, statistically significant. 
Figure 1

The volcano plot

The red spots represent 202 up-regulated miRNAs and the green spots represent 21 downregulated miRNAs (blue imaginary lines represent Fold change: \pm 2 and P-value: 0.05 ). 


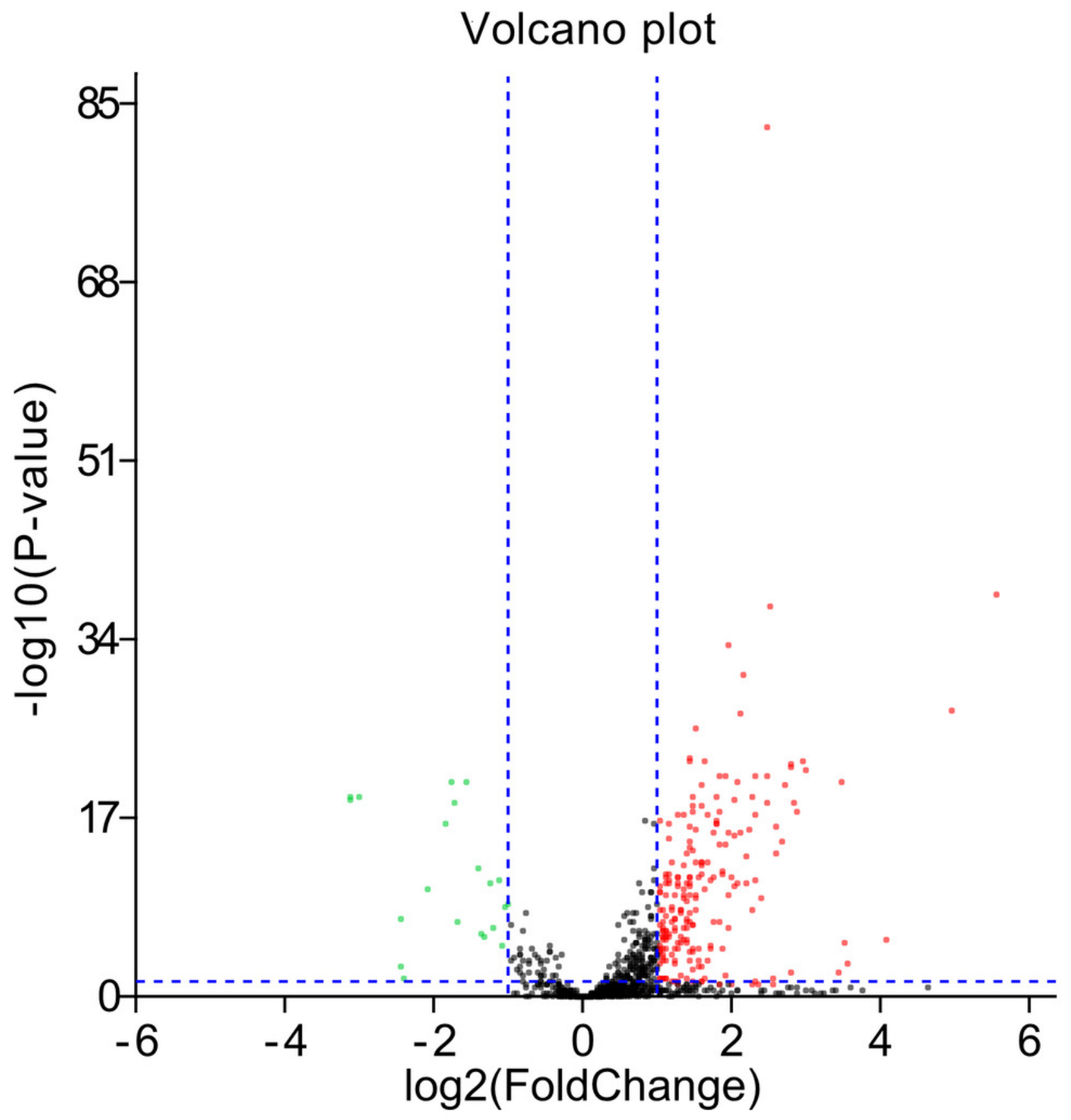


Figure 2

The expression of miR-383-5p in GC tissues and cells.

A: miRNA-Seq data indicated that miR-383-5p was down-regulated in GC tissues; B, C: qRTPCR showed that miR-383-5p was significantly down-regulated in GC cells and tissues. * $\mathrm{P}<0.05$ compared with the control group (GES-1 or normal tissues).

A

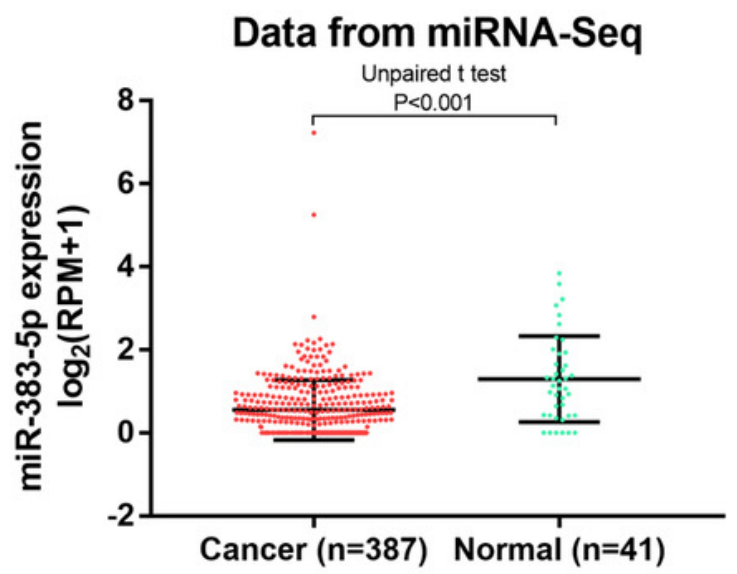

B

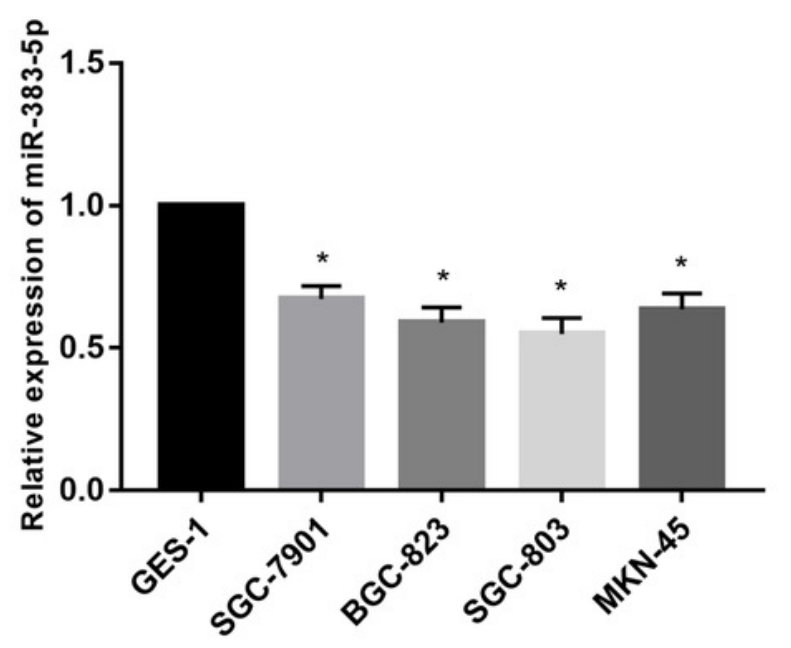

C

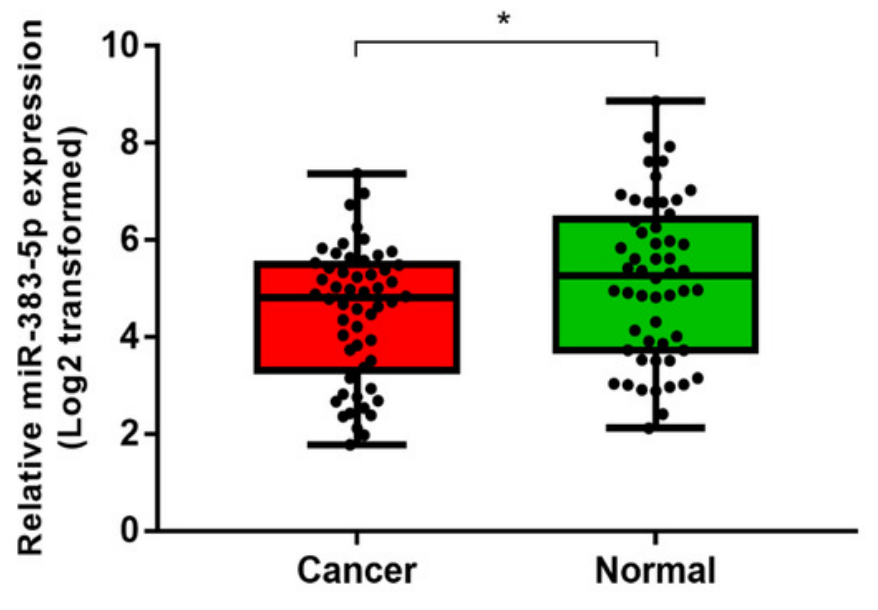


Figure 3

The Kaplan-Meier overall survival curve

The group with low miR-383-5p expression had a significantly less survival time (median survival time 21.1 months) than that with high expression (46.9 months).

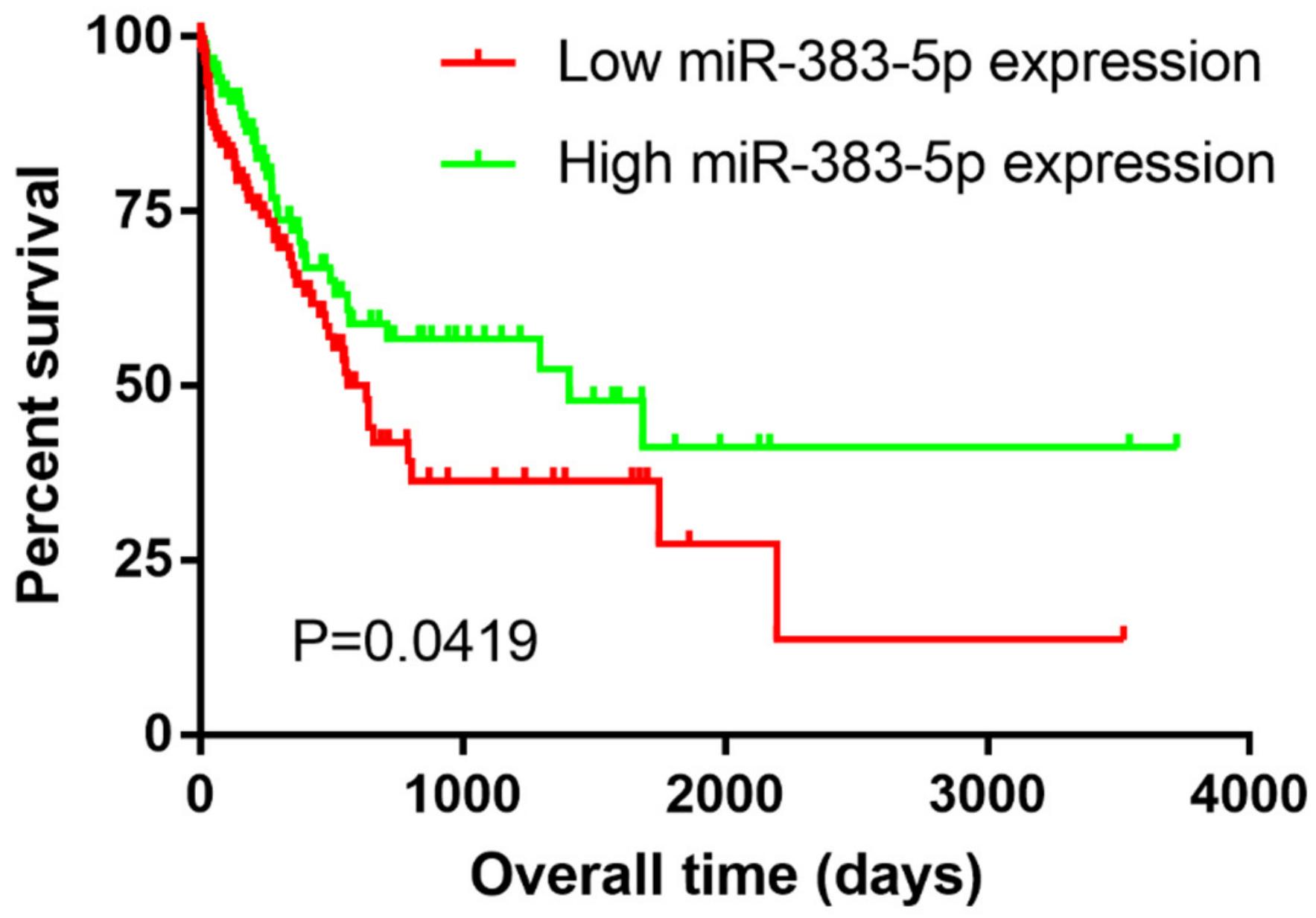




\section{Figure 4}

The effect of miR-383-5p on the proliferation and migration of GC cells

A-H: miR-383-5p mimics inhibited GC cells migration; I,J: miR-383-5p mimics inhibited GC cells proliferation. $* \mathrm{P}<0.05$ compared with NC group.

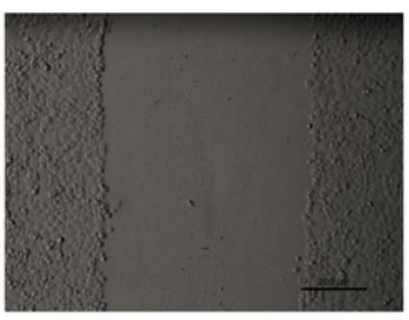

MGC-803 NC 0h

A

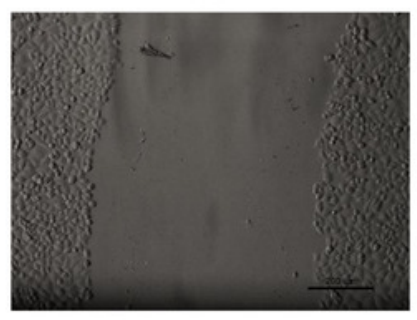

MGC-803 mimics 0h

$\mathbf{E}$

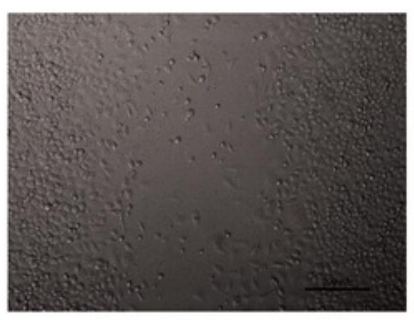

MGC-803 NC 48h

B

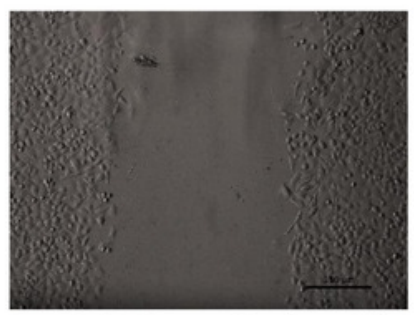

MGC-803 mimics 48h

F

MGC-803

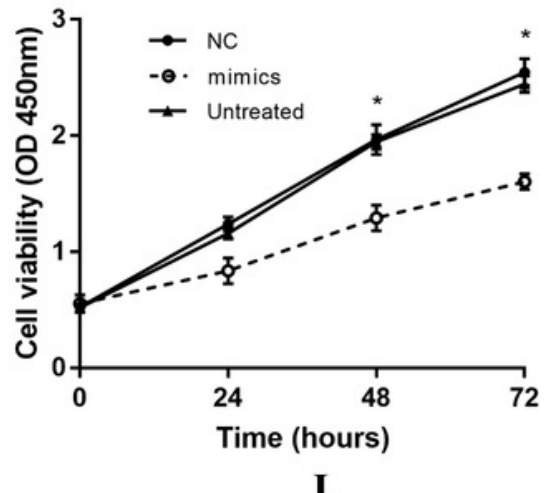

I

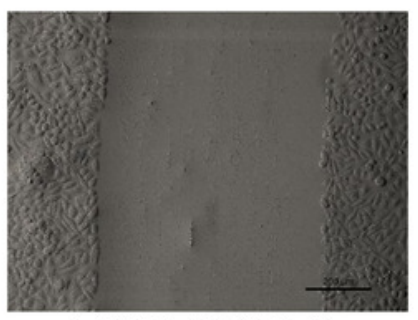

MKN-45 NC 0h

C

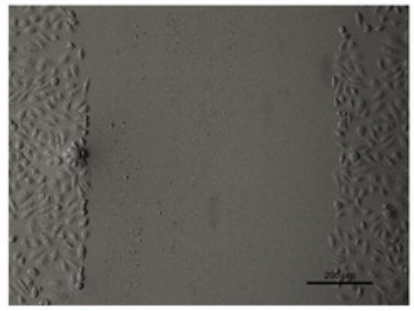

MKN-45 mimics 0h

G

MKN-45

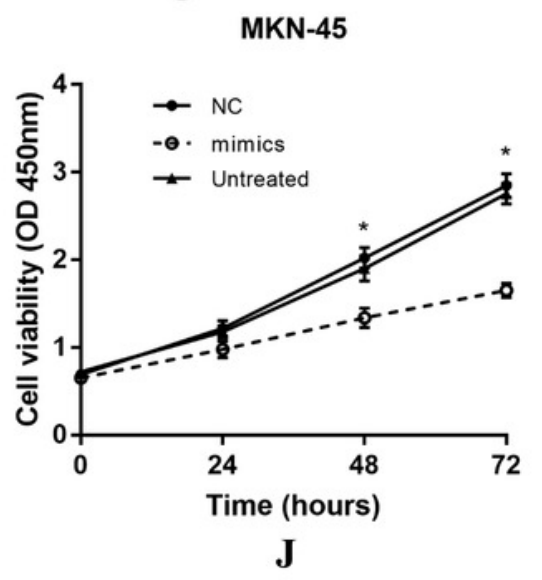

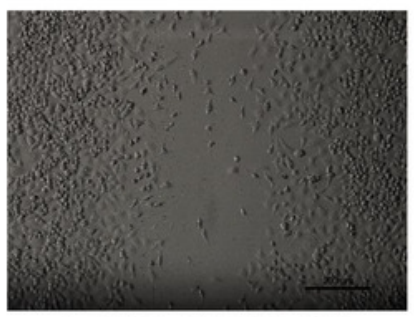

MKN-45 NC 48h

D

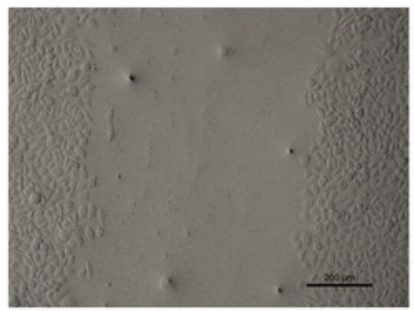

MKN-45 mimics 48h H 
Figure 5

The venn plot of target genes and functional enrichment analysis

A: A total of 49 consensus genes were obtained from TargetScan, miRDB, and starBase websites; B: Biological pathway analysis revealed that these genes participated in PI3K, mTOR, c-MYC, TGF-beta receptor, VEGF/VEGFR and E-cadherin signaling pathways.

A

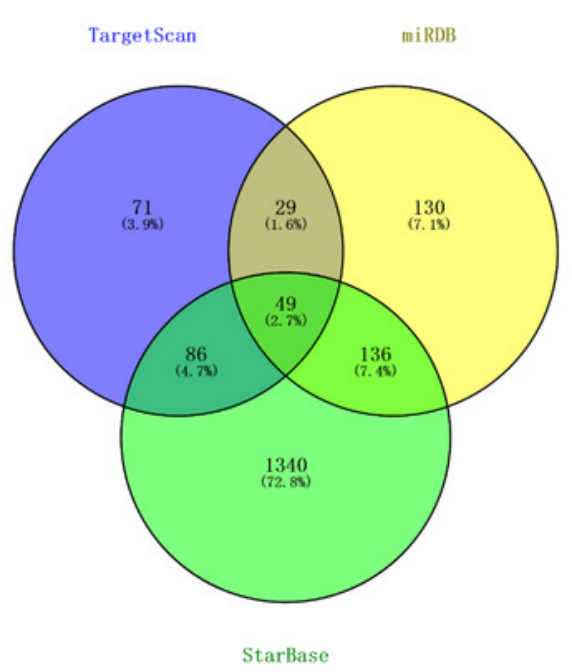

B

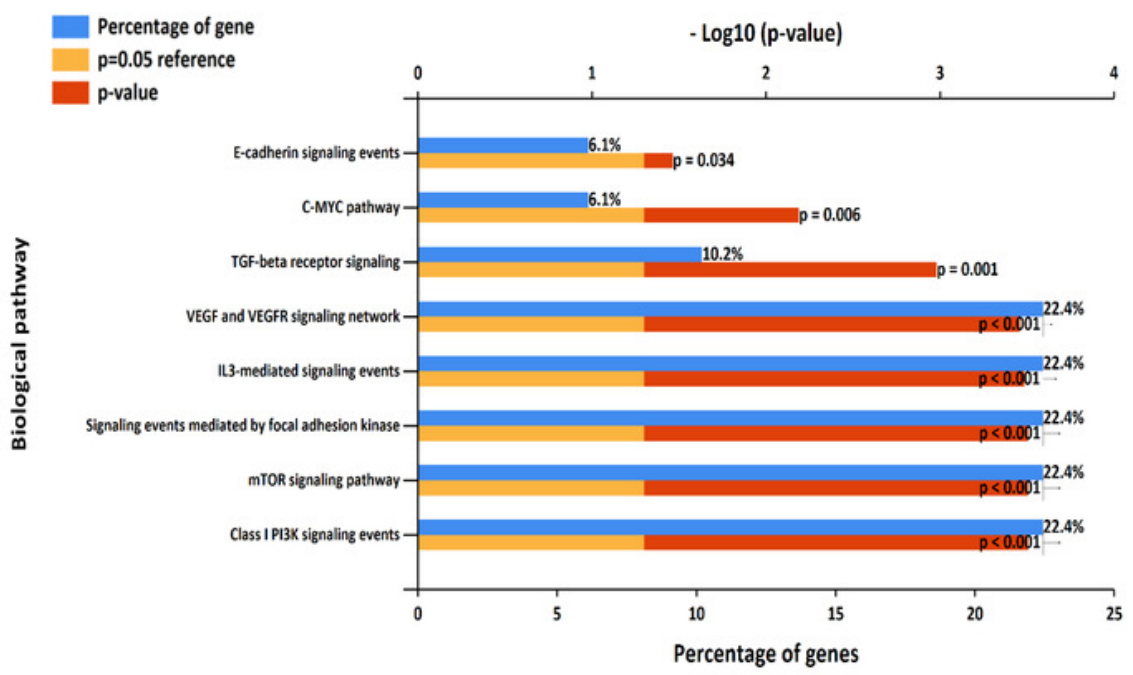




\section{Figure 6}

miR-383-5p directly targets LDHA

A: The predicted miR-383-5p binding sites on the 3'-UTR of LDHA; $B$ : The data based on starBase project showed that expression of miR-383-5p was negatively correlated with target LDHA ( $r=-0.203)$; C: Luciferase reporter assay indicated that the luciferase activity in wildtype LDHA-3'UTR group was significantly decreased by the miR-383-5p mimics. $* P<0.05$.

A Binding Site of hsa-miR-383-5p on LDHA:

\begin{tabular}{|l|l|l|l|}
\hline BindingSite & $\uparrow$ & Class & Alignment \\
\hline chr11:18428908-18428914[+] & $\uparrow$ & 7 mer-m8 & Target: 5' uguuguccuUUUUAUCUGAUCu 3' \\
\hline & & & I: : : I। I।। I। \\
\hline
\end{tabular}

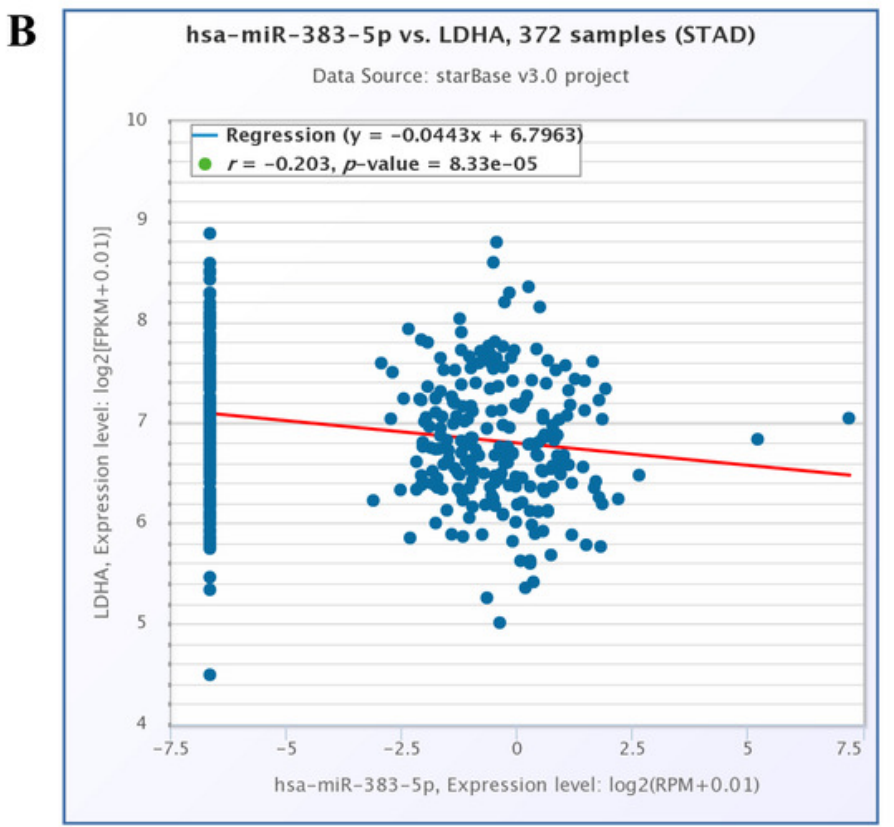

C

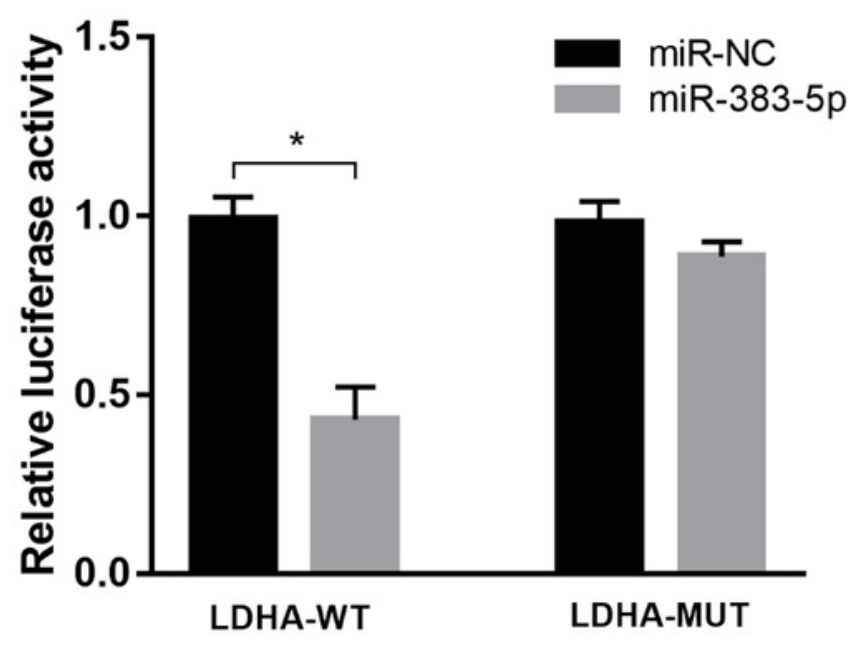


Table $\mathbf{1}$ (on next page)

Sequence of primers used for PCR.

Sequence of primers used for PCR. 
1 Table 1. Sequence of primers used for PCR.

\begin{tabular}{ll}
\hline Name & Sequence $\left(\mathbf{5}^{\prime} \mathbf{-} \mathbf{3}^{\prime} \mathbf{)}\right.$ \\
\hline mR-383-5p (RT) & GTCGTATCCAGTGCGTGTCGTGGAGTCGGCAATTGCACTGG \\
& ATACGACAGCCAC \\
mR-383-5p (forward) & GGGAGATCAGAAGGTGATTGTGGCT \\
mR-383-5p (reverse) & CAGTGCGTGTCGTGGAGT \\
U6 (forward) & CTCGCTTCGGCAGCACA \\
U6 (reverse) & AACGCTTCACGAATTTGCGT \\
\hline
\end{tabular}

2 


\section{Table 2 (on next page)}

42 DEMs identified between GC and adjacent normal tissues.

42 DEMs identified between GC and adjacent normal tissues. 
1 Table 242 DEMs identified between GC and adjacent normal tissues.

\begin{tabular}{lll|lll}
\hline Down-regulated & logFC & P value & Up-regulated & logFC & P value \\
\hline miR-1-3p & -3.13 & $1.56 \mathrm{E}-19$ & miR-196a-5p & 5.55 & $3.01 \mathrm{E}-39$ \\
miR-133a-3p & -3.13 & $9.66 \mathrm{E}-20$ & miR-196b-5p & 4.94 & $3.33 \mathrm{E}-28$ \\
miR-133b & -3.03 & $9.52 \mathrm{E}-20$ & miR-767-5p & 4.05 & $3.28 \mathrm{E}-06$ \\
miR-802 & -2.46 & 0.000768 & miR-552-3p & 3.55 & 0.000579 \\
miR-490-3p & -2.46 & $2.63 \mathrm{E}-08$ & miR-105-5p & 3.49 & $7.65 \mathrm{E}-06$ \\
miR-1265 & -2.43 & 0.014022 & miR-135b-5p & 3.46 & $2.25 \mathrm{E}-21$ \\
miR-204-5p & -2.10 & $3.67 \mathrm{E}-11$ & miR-767-3p & 3.44 & 0.003757 \\
miR-145-5p & -1.87 & $3.04 \mathrm{E}-17$ & miR-194-5p & 2.97 & $2.20 \mathrm{E}-22$ \\
miR-139-3p & -1.76 & $2.84 \mathrm{E}-21$ & miR-200a-5p & 2.94 & $2.40 \mathrm{E}-23$ \\
miR-145-3p & -1.75 & $2.37 \mathrm{E}-19$ & miR-192-5p & 2.87 & $1.71 \mathrm{E}-18$ \\
miR-129-5p & -1.70 & $4.41 \mathrm{E}-08$ & miR-200a-3p & 2.81 & $2.26 \mathrm{E}-19$ \\
miR-139-5p & -1.59 & $2.49 \mathrm{E}-21$ & miR-200b-3p & 2.80 & $1.08 \mathrm{E}-22$ \\
miR-30a-3p & -1.41 & $4.25 \mathrm{E}-13$ & miR-141-5p & 2.79 & $5.05 \mathrm{E}-23$ \\
miR-490-5p & -1.40 & $6.09 \mathrm{E}-07$ & miR-1269a & 2.78 & 0.003274 \\
miR-551b-3p & -1.32 & $1.73 \mathrm{E}-06$ & miR-183-5p & 2.71 & $4.27 \mathrm{E}-21$ \\
miR-143-3p & -1.27 & $1.05 \mathrm{E}-11$ & miR-194-3p & 2.64 & $1.03 \mathrm{E}-15$ \\
miR-486-5p & -1.22 & $2.70 \mathrm{E}-07$ & miR-429 & 2.58 & $2.15 \mathrm{E}-14$ \\
miR-29c-3p & -1.14 & $7.86 \mathrm{E}-12$ & miR-141-3p & 2.57 & $5.25 \mathrm{E}-17$ \\
miR-383-5p & -1.12 & $1.13 \mathrm{E}-05$ & miR-675-5p & 2.55 & 0.011142 \\
miR-195-3p & -1.06 & $1.83 \mathrm{E}-09$ & miR-146b-5p & 2.50 & $4.41 \mathrm{E}-38$ \\
\hline
\end{tabular}


Table 3 (on next page)

Association between the genes and clinical features.

Association between the genes and clinical features. 
1 Table 3. Association between miR-383-5p expression and clinical features.

\begin{tabular}{lccccc}
\hline \multirow{2}{*}{ Variables } & \multicolumn{2}{c}{ MiR-383-5p expression } & Total & Pearson & P value \\
\cline { 2 - 3 } & Low (n,\%) & High (n, \%) & samples & $\boldsymbol{r}$ & \\
\hline Age & & & & & \\
$<60$ & $11(17.1)$ & $12(13.4)$ & 23 & -0.037 & 0.783 \\
$\geq 60$ & $16(28.0)$ & $15(41.5)$ & 31 & &
\end{tabular}

Gender

Male

$17(32.9) \quad 13(28.1) \quad 30$

Female

$10(25.6) \quad 14(13.4)$

$0.149 \quad 0.273$

Tumour size

$\leq 5 \mathrm{~cm}$

$>5 \mathrm{~cm}$

$\begin{array}{ccc}5(9.8) & 14(15.9) & 19 \\ 22(48.8) & 13(25.6) & 35\end{array}$

$-0.349 \quad 0.010^{*}$

Lymph node metastasis

Negative

Positive

$15(45.1) \quad 9(22.0)$

24

$\begin{array}{ll}-0.224 & 0.100\end{array}$

TNM stage

$\begin{array}{lccccc}\text { I + II } & 13(28.0) & 20(15.9) & 33 & -0.266 & 0.051 \\ \text { III + IV } & 14(30.5) & 7(25.6) & 21 & & \end{array}$

\section{Differentiation grade}

Well and moderate

$6(17.1) \quad 16(18.3) \quad 22$

Poor

$21(41.5)$

$11(23.2)$

$-0.377 \quad 0.006^{*}$

$2 * P<0.05$, statistically significant. 